\section{TURNING THE SPOTLIGHT ON PLAQUE}

New to GC UK's product range is Tri Plaque ID Gel. As the name suggests Tri Plaque ID Gel allows you and your patient to identify areas of plaque in three easy steps.

GC's Tri Plaque ID Gel quickly identifies new, mature and acid producing biofilms. This unique gel also highlights exactly where the bacteria are most active by disclosing the acidic $\mathrm{pH}$. This additional information will be a great help in your daily practice to motivate your patients to improve their oral hygiene.

The Gel is colour coded for easy identification:

- Blue/purple - old plaque (more than 48 hours)

- Red/pink - newly formed plaque

- Light blue - high risk plaque.

Tri Plaque ID Gel encourages patients to be more precise with their tooth brushing technique. Once diagnosis is complete simply brush the teeth to clean them.

For further information contact GC UK on 01908218999.

\section{SECURE PAYMENT PROCESSING}

Anderson Zaks provides customers with highly reliable, fast and secure payment processing services. Customers benefit from Anderson Zaks' flexibility, personalised customer care and over 16 years' experience.

Anderson Zaks addresses the needs of businesses in all shapes and sizes, including those new to card acceptance. Their RedCard Processing Services handle transactions from over 15,000 sites. Data security is a top priority; data centres are PCI Level 1 certified and Red-
Card Applications have been PA-DSS validated. Anderson Zaks maximise your choice of acquirers, devices, cards, transaction types and value added services and reduce the amount of time it takes to integrate systems.

For informed advice and a reliable payment service, telephone 01344 317910, email info@andersonzaks.com or visit www.andersonzaks.com.

\title{
PEPPERMINT, SPEARMINT AND CINNAMON TOOTHPASTE
}

Xylitol's natural properties make it a unique sweetener - it prevents bacteria adhering to teeth and gums, therefore minimising plaque formation and improving the health of the oral cavity.

Spry is one of the UK's leading providers of high-quality xylitolbased dental care products, including dental gums, mints, toothpastes, dental floss, and mouth rinses.

Spry's fluoride-free toothpastes contain more than 25\% xylitol, are rich in calcium and low in alcohol. The low-abrasive formula for anti-plaque action is gentle on enamel. For adults, they come in three flavours: peppermint, spearmint and cinnamon. In addition, the cinnamon toothpaste, as a non-menthol flavour, is especially suitable for those on homeopathic remedies.

www.anyone4tea.com

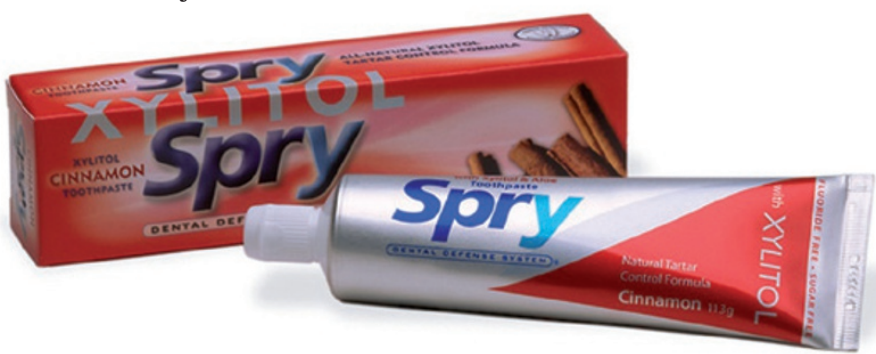

\section{FLEXIBLE ROUTES TO MOUTH CANCER SCREENING}

In a bid to encourage as many dental practices as possible to sign up to the newly launched Mouth Cancer Screening Accreditation Scheme, the Mouth Cancer Foundation has introduced a £20 per month direct debit option to prospective members. There is also the choice of an annual upfront payment of $£ 200$.

Dental practices that join the Mouth Cancer Screening Accreditation Scheme are accredited by the Mouth Cancer Foundation when they demonstrate a visible commitment to increasing public awareness of mouth cancer screening to all patients and establish a documented referral pathway with a local specialist department. Dentists must meet certain criteria and routinely participate in oral cancer screening. There is a quick, detailed examination that takes just two minutes and can save lives.

For the membership fee dental teams will learn how to perform this exam and will have access to a dedicated section of the charity website and a free hour of continuing professional development (CPD). There are also professional development and training modules suitable for all members of the practice team. The membership fee also includes a certificate and plaque for the practice to display.

Dental practices joining the Screening Accreditation Scheme are required to pay a fee to cover the charity's costs for administering the scheme. The Direct Debit facility is being managed by DPAS.

To find out more email info@ themouthcancerfoundation.org or telephone 01924950950.

\section{CARIES INFILTRATION THERAPY}

Icon is DMG UK's innovative caries infiltration therapy. It represents a breakthrough in micro-invasive technology that reinforces and stabilises demineralised enamel without drilling or sacrificing healthy tooth structure. It is the first product to bridge the gap between prevention (fluoride therapy) and caries restoration. Icon's microinvasive infiltration technology can be used to treat smooth surface and proximal carious lesions up to the first third of dentine. In just one visit Icon can arrest the progression of early enamel lesions and remove carious white spot lesions.

See www.drilling-no-thanks. co.uk and www.dmg-dental.com. 